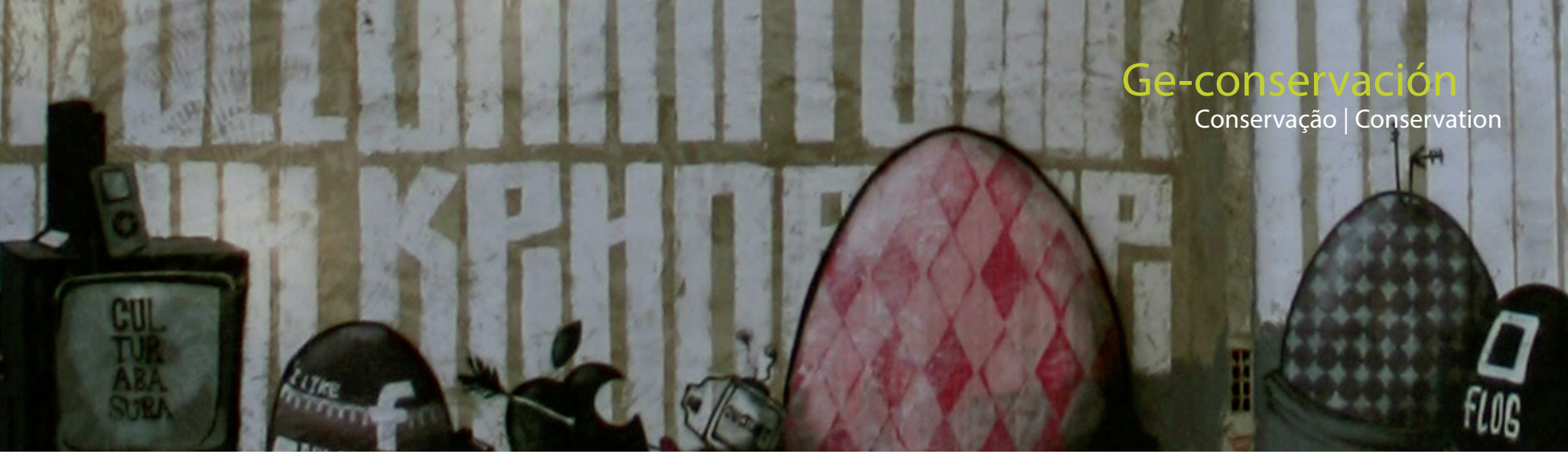

\title{
Recreación virtual del arte urbano
}

\author{
María del Mar Vázquez de la Fuente
}

Resumen: El presente texto versará sobre la complicidad que algunos videojuegos guardan con el arte urbano. Para ello, se va a recopilar diversos títulos de juegos para distintas plataformas que representan el acto de la acción del grafiti y, en algunos casos, incluso permiten al jugador la posibilidad de crear sus propias obras. Además, en una segunda parte del artículo, se tratará el tema de la geolocalización del arte urbano y la recreación virtual de la experiencia in situ como es el caso del juego Pokémon Go y su vinculación con estas creaciones ubicadas en el espacio público.

Palabras clave: videojuegos, arte urbano, recreación, experiencia

\section{Virtual recreation of street art}

Abstract: This text will focus about the complicity of some video games with Street Art. For that, will be collected sundry titles for different platforms that represent the act of making grafiti and, in some cases, even allows the player make their own works. Futhermore, in a second part of the article, will be treated the topic of geolocation of Street Art as a virtual recreation of the experience in situ such as Pokémon Go and it's vinculation with this creations located in the public space.

Key words: video games, street art, recreation, experience

\section{Introducción}

A la hora de analizar el grosso de los videojuegos que se han creado actualmente, se puede apreciar que muchos de ellos se han enfocado hacia la simulación de la realidad, evolucionando hasta llegar al actual estado de boom mundial frente a la realidad virtual'. Los videojuegos, en cierto modo, siempre han perseguido este objetivo del realismo, generando simuladores de vuelo, conducción, shooters en primera persona, etc.; y cabría preguntarse hasta qué punto se ha extrapolado estas simulaciones al arte urbano. A través de los siguientes ejemplos la idea es ver como algunos videojuegos permiten al jugador sumergirse en la experiencia de la creación de arte urbano mediante la simulación de esta actividad.

\section{Marc Eckō's Getting Up: Contents under pressure}

Este primer ejemplo, diseñado por Marc Echō, es el videojuego más conocido de la temática de arte urbano. En el título ya se aprecia que el juego está muy unido a la cultura urbana, ya que getting up significa, en esta situación, la capacidad de darse a conocer en el ámbito urbano; así como contents under pressure hace alusión al etiquetado de los aerosoles en los que se indica que el contenido está bajo presión (que además funciona en este caso como un símil a la represión política que sufre la ciudad donde se desarrolla la acción, siendo el elemento narrativo principal).

La trama gira en torno al ascenso de Trane, el protagonista, hasta convertirse en una estrella del grafiti, siendo instruido 
durante su aventura por artistas urbanos conocidos como Cope2, FUTURA, OBEY, Seen, Smith y T-kid 170. La elección de estos personajes reales no es algo dejado al azar, ya que cada uno enseña a Trane una habilidad que, por sus destrezas, les han sido adjudicadas a estos artistas: habilidad para marcar el sistema de metro, pintar murales, el empleo de adhesivos, bombardear vagones, sigilo...

Este juego ha generado mucha polémica, ya que fue censurado en Australia y en Inglaterra por la Asociación Anti-Graffiti. Marc Echō, en una entrevista en el periódico neoyorkino Metro, dijo que este conflicto no es debido solo a la visión criminalizada que se tiene sobre el grafiti, sino además a una desconexión generacional con las tecnologías².

La relevancia de Marc Echō's: contents under pressure reside en que este juego ha sido concebido como el primer simulador de grafiti y hace referencia a una serie de artistas urbanos que otorgan a este título un grado importante de complicidad con este tipo de creaciones, además de ser uno de los primeros proyectos que se acercaron al arte urbano, y funciona a modo de documento de algunas técnicas empleadas así como de algunos de sus artistas más reseñados. Tanto es así que Jota Mayúscula, doblador de voz de Trane, afirma que lo que le llevó a aceptar la participación en el juego fue el respeto con el que trabajaron hacia la cultura del grafiti y el Hip Hop, además de reflejar la escena del arte urbano correspondiéndose a un cien por ciento de la realidad ${ }^{3}$.

\section{WiiSpray: aerosol cableado, pintura digital}

Como resultado del proyecto Information places de 2007, los estudiantes Martin Lihs y Frank Matuse, pertenecientes al Media Department de la Bauhaus University Weimar, crearon un dispositivo que, unido al mando de la consola de Nintendo Wii, permite la posibilidad de usar un objeto visualmente parecido a un aerosol, ofreciendo además una función similar de manera digital.

Junto a este hardware o dispositivo, se ha elaborado un programa o software que funciona a modo de paleta virtual, de tal forma que se pueden elegir entre diversos colores, permitiendo mezclarlos para crear numerosos tonos personalizados. A esta libertad cromática se le añade la posibilidad de poder elegir el tipo de aerosol que el jugador necesita en cada momento para calibrar a su gusto el tipo de trazo que realiza, así como el tipo de boquilla que se precisa para cada efecto. Tal es el empeño de alcanzar el realismo que, entre las opciones que ofrece WiiSpray, se pueden emplear plantillas personalizadas.

La idea más interesante de este proyecto es la posibilidad de poder realizar unos primeros acercamientos al arte urbano y poder usarlo como una herramienta de experimentación y de toma de contacto con los materiales que se emplean habitualmente en estas creaciones. Además, sirve como una ayuda en el estudio previo a la realización de una obra por parte de los artistas, para así poder visualizar qué resultados obtendrían.

\section{GRAFITE VIRTUAL: de la calle a los eventos sociales}

Actualmente, la sociedad ha ido aceptando con el paso del tiempo la cultura del arte urbano, ya que, como dice Aldo Iván Gómez en su artículo, El Graffiti como fuente de inspiración en la industria de la moda, afirma: "La filosofía delos primeros años del graffiti ha ido cambiando paulatinamente debido a la inmersión de este como un producto de moda. En un principio se busca la discreción, donde se debía de realizar bajo el anonimato. Ahora las marcas están al pendiente de cuál es el graffitero más sobresaliente del momento para poder realizar colaboraciones con estos. Se dieron cuenta de que el graffiti vende ${ }^{\prime \prime 4}$. Es decir, estas expresiones artísticas cada vez están ganando más aceptación, llegando a calar incluso en grandes marcas como es el caso del previamente entrevistado artista taiwanés Ano, que colaboró con Nike y Adidas en varios eventos ${ }^{5}$.

Un buen ejemplo de la aceptación y del interés de las grandes marcas por el arte urbano, en concreto en su versión digital, es Grafite Virtual. En la promoción de este simulador ya se advierte que la idea de sus creadores, de origen brasileño, procede por el interés del grafiti de su país, ya que afirman que ese estilo es uno de los más reconocidos del mundo. Usando este pensamiento casi a modo de premisa, sus creadores ofrecen una funcionalidad muy similar al citado WiiSpray, pero en su defecto, Grafite Virtual está enfocado más a su uso en eventos sociales que a un juego para el hogar, como sería el caso del proyecto para Nintendo.

Este simulador ofrece una gran cantidad de colores, formatos, opciones e incluso accesorios para su utilización, aunque $-y$ aun presumiendo de su novedad a nivel nacional y mundial-el dispositivo con el que se realizan las creaciones también es similar al formato de WiiSpray, imitando a un aerosol; pero en este caso funciona con unas latas originales adaptadas con sensores y tecnología de infrarrojos. Como resultado, Grafite Virtual es una herramienta visualmente muy atractiva que ha sabido mostrar que el arte urbano tiene potencial a la hora de presentar a una empresa o a un producto como un elemento muy llamativo e interactivo. Son muchas las marcas y empresas que han contado con los servicios de esta herramienta para promocionarse, entre ellos Google, Facebook, SAP, Natura, Yahoo, Coca-cola, Chanel o Mercedes-Benz . [figura 1].

La relevancia de este sistema es que hace ver que el arte urbano tiene un gran impacto social, ya que es una actividad rápida y fresca, tan dinámica como la tecnología y el videojuego, permitiendo todo ello dar libertad a la creatividad, como en los casos de los escaparates de Chanel o la feria de Mercedes Benz, en los que los transeúntes se convierten en artistas improvisados y, como con los materiales reales, pueden dejar una huella personalizada de esta acción. 


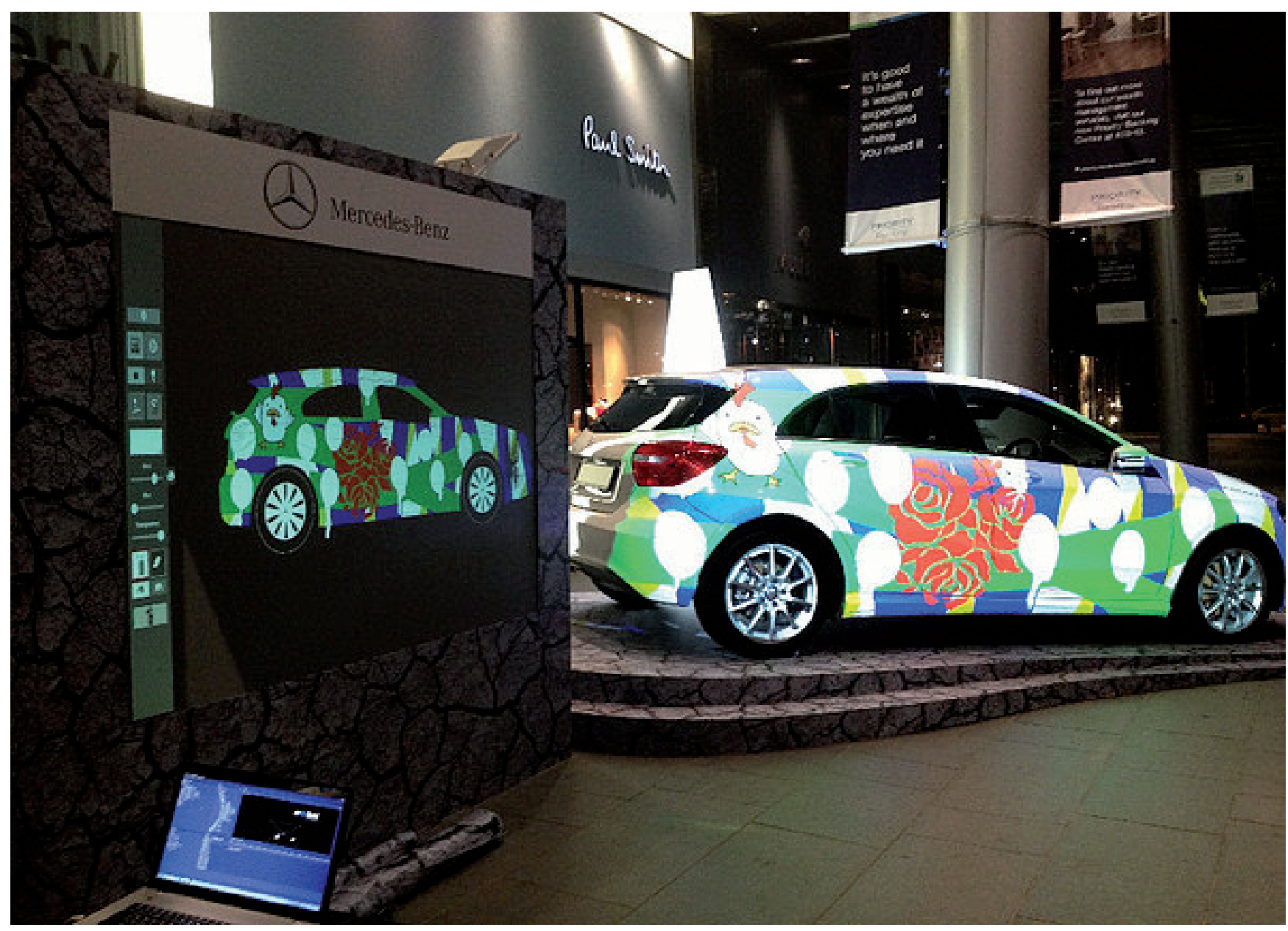

Figura 1.-Exposición de Mercedes Benz usando Grafite Virtual. Contenido perteneciente a: http://www.grafitevirtual.com.br/grafitedigital

\section{Kingspray graffiti simulator: La experiencia en realidad virtual}

Gracias a la evolución tecnológica, el nuevo punto de mira de los videojuegos se centra en la realidad virtual. Esta herramienta se ha aplicado a distintos géneros dentro de los videojuegos, como ya se ha adelantado previamente; $y$, con este ejemplo, se puede ver que ha llegado a aproximarse al arte urbano.

Este simulador ofrece unas características similares a las citadas en los ejemplos de Grafite virtual o WiiSpray, pero su novedad está en que ha sido pensado y desarrollado para las gafas de realidad virtual, unos periféricos que sirven para dar sensación de inmersión en un escenario imaginario.

En este caso, la acción se desarrolla en un entorno urbano en el cual se puede elegir la zona sobre la que se quiere realizar la intervención. Kingspray graffiti simulator es un juego que ha querido llevar el realismo de la experiencia al máximo, ya que entre las configuraciones puedes elegir qué hora del día quieres que sea y, entre otros efectos, si aplicas mucha cantidad de producto se puede dar un efecto dropping muy realista.
Todo esto muestra cómo el arte urbano es de gran interés para el mundo del videojuego y los simuladores, ya que proporciona experimentación de una acción "ilegal" dentro de un entorno idealizado y, además, se podrán conservar las obras y compartirlas gracias a las funciones relacionadas con las redes sociales. [Figura 2].

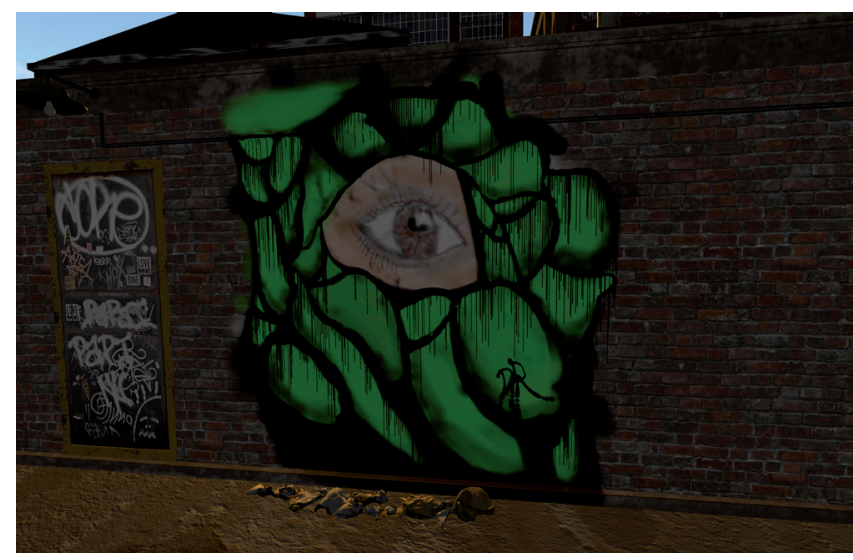

Figura 2.- Proyecto realizado con Kingspray graffiti simulator. Contenido perteneciente a: http://graffitisimulator.com/ kingspray-35/ 


\section{Augmented reality creator: Generador en App}

Además de juegos y simuladores, como los anteriormente citados, podemos encontrar en otras plataformas diversos recursos igualmente interesantes, siendo en este caso para teléfonos de nueva generación o smartphones: Augmented reality creator.

Diseñada para el sistema operativo iOS, Augmented reality creator combina un editor de realidad virtual con redes sociales, aunque, como en el arte urbano, ofrecen la posibilidad de publicar en la librería de la propia aplicación con total anonimato. Es reseñable que juega con la perspectiva, en tiempo real, en el emplazamiento en el que se encuentre el jugador, quien es ayudado al realizar el grafiti por un personaje animado llamado Max Morrison, al cual se puede personalizar. La aplicación, como novedad, ofrece la posibilidad de crear arte urbano en cualquier parte y momento, usando como soporte lo que quiera en ese momento el jugador.

Augmented reality creator, además, en su página web, va acompañado de unas palabras de su creador donde deja clara su percepción del arte urbano:

"El grafiti de spray no es aceptado socialmente, pero no te preocupes por ser cogido con un bote de aerosol (virtual) en las manos- Max Morrison se llevará la culpa. Max es el tipo adecuado para el trabajo; es un marginado, no es extremadamente bien aceptado en una vida diaria medida por los estándares tradicionales de una vida exitosa. Él se siente orgulloso de sí mismo en ser libre y extremadamente intransigente. A él no le importa. Si piensa o siente que hay algo que decir, lo dirá. No solo lo dirá, lo grafiteará, y cuanto más atractivo el soporte, mejor. El edificio del parlamento, una sala de ópera, un colegio, detrás de una chica guapa, si la superficie se ciñe a su propósito, es rociado con el spray. Su propósito en vida es dibujar grafitis en todos los edificios famosos en el mundo. Los usuarios tienen que ser animados a ser como Max y grafitear lo que sea que tengan en mente en ese momento, ser creativos, divertidos, y controvertidos [...]. No hay límite para la creatividad de los usuarios, elaborando mensajes potentes con un excelente potencial viral." ${ }^{\text {. }}$.

De estas palabras podemos extraer como la percepción de su creador es la de un arte urbano muy reivindicativo que bombardea la ciudad con mensajes polémicos. Por ello, el interés de esta aplicación reside en la idea con la que fue concebida: un lugar donde compartir pensamientos, donde poder expresarse libremente sin censura. En definitiva, quiere mimetizar la libertad creativa del arte urbano, dando cabida a todas las opiniones y guardando todas esas intervenciones ficticias en una misma aplicación.

\section{Pokémon Go y la geolocalización del arte urbano}

En julio de este año se estrenó el conocido juego para plataformas móviles Pokémon Go, el cual experimenta con la realidad inmersiva para poder capturar las criaturas creadas por la compañía Game Freak.

El objetivo se resume en ser el mejor entrenador de pokémon, y para ello, hay que obtener materiales en unos lugares llamados "pokeparadas" para poder capturar y fortalecer a las criaturas. Estas zonas denominadas así, son emplazamientos de interés general vinculados al entramado urbanístico (estatuas, tiendas, edificios...). Es justo en este punto en el que el arte urbano se convierte en una herramienta muy importante para Pokémon Go. Al entrar en contacto con el juego, destaca que muchos de los puntos de interés están relacionados con grafitis. Esto deja un tema abierto: la obra como punto de encuentro de los jugadores y el conocimiento del entorno. Por un lado, Pokémon Go ha vinculado estos puntos de encuentro a las "pokeparadas", lugar donde es habitual observar a personas congregadas en torno a esa zona, geolocalizado que cerca existe un grafiti. Esto, además

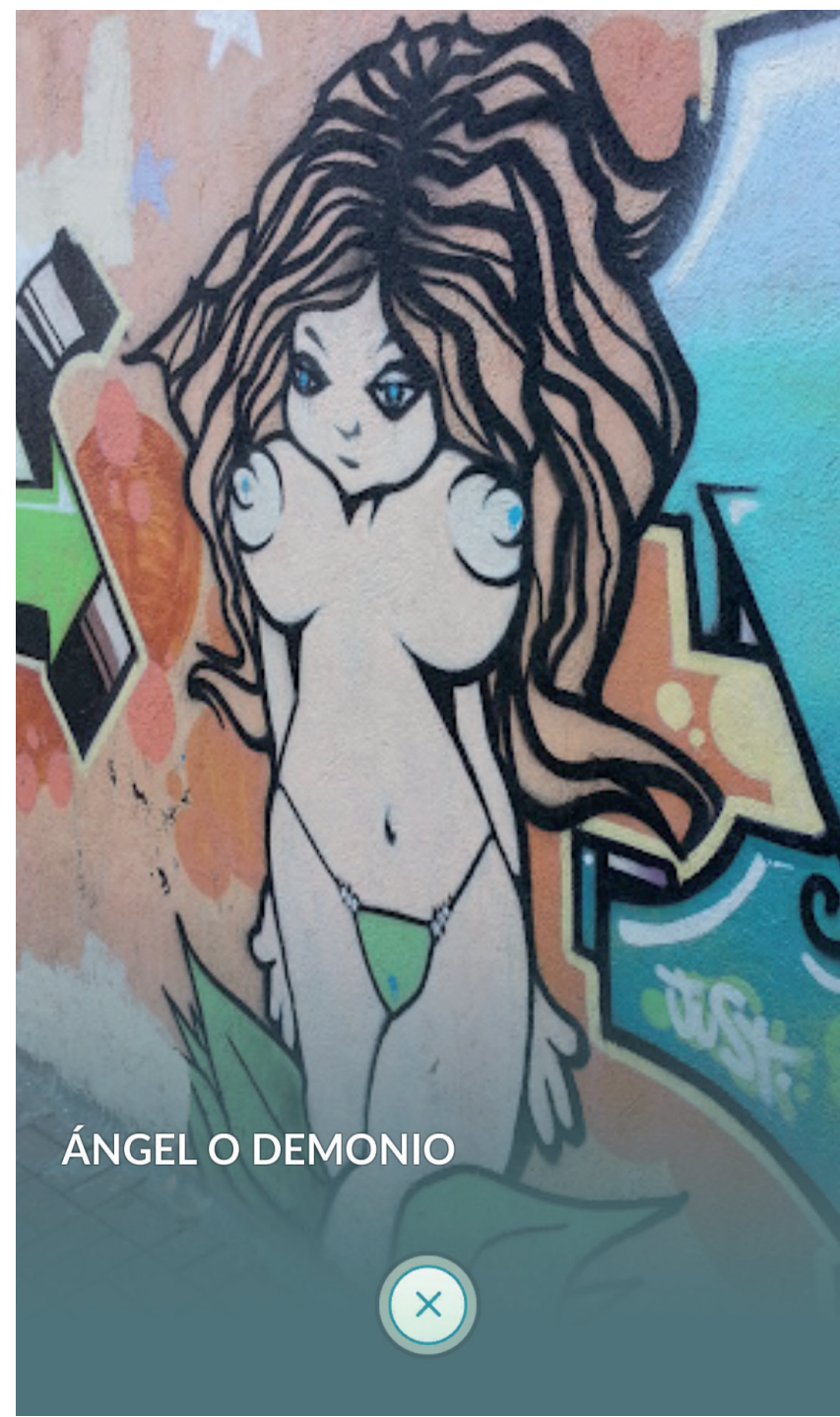

Figura 3.- Intervención denominada "Ángel o Demonio". Captura realizada por: María del Mar Vázquez 
de acrecentar la socialización, crea un conocimiento del propio entorno y de intervenciones que, quizás, de otra forma habrían pasado desapercibidas. Por otro lado, no habría que pasar por alto que esta predilección hacia el arte urbano funciona en cierto modo como una forma de documentación. Observando la imagen propuesta por la aplicación podemos acudir posteriormente a su ubicación y proceder a evaluar sus cambios. Este asunto es especialmente interesante en este campo de la conservación, ya que facilita la documentación. El caso más concreto es el de una intervención llamada en Pokémon Go "Angel o Demonio". Este grafiti, ubicado en el barrio de Vallecas, ha sido empleado como punto de interés de una "pokeparada". Transcurrido el tiempo, ese grafiti fue eliminado, pero en el juego se mantiene su presencia. [Figura 3].

Lachlan MacDowall en su artículo For lovers of graffiti, Pokémon Go is old hat ${ }^{7}$ comenta que esta idea de Pokémon Go no es nueva, ya que la gente, con anterioridad a este juego, procedían a capturar imágenes antes de que desapareciera la obra de artistas reconocidos como Banksy. Como él mismo apunta, es reseñable que este juego pone en alza a obras más y menos conocidas como puntos de interés con una recompensa para el jugador, además de la propia de poder transitar la ciudad y conocer su arte urbano.

\section{Conclusiones}

Tras estos ejemplos citados en el presente texto, cabría destacar que solo son una pequeña porción de todos los juegos que se podrían analizar, ya que hay otros ejemplos existentes; pero casi todos en la misma línea de los que aquí se han recogido y analizado.

Como se ha podido observar en este artículo, no solo se ha llevado el arte urbano al videojuego, tanto como objetivos a cumplir de la narrativa, como escenarios, etc., sino que ha llegado al ámbito tecnológico del hardware para convertirse en una herramienta que se encuentra entre el ocio y el diseño gráfico.

Algunos juegos y simuladores se presentan como alternativas experimentales muy útiles para preparativos previos a las intervenciones $y$, además, sirven como un elemento didáctico para acercar la cultura del arte urbano. Así mismo, otros juegos crean unos ambientes que sumergen al jugador en un contexto adaptado a la realidad, donde pueden meterse en la situación y en el rol de los artistas urbanos.

Por todo ello, habría que analizar todos estos simuladores como una herramienta útil desde el punto de vista creativo; pero, además habría que estudiar la posibilidad de que sirvan como ayuda a la documentación de estas obras, como es el caso de Pokémon Go en su geolocalización.
De otra forma, todo este artículo, junto a las investigaciones realizadas anteriormente, deja entrever aún más la importancia de la relación del arte urbano con la tecnología y, en este caso en concreto, con el videojuego. Por un lado, no se ha podido obviar que muchos artistas se han inspirado en los códigos visuales y personajes como autoproclama o muestra de su influencia por los videojuegos. Por otro, los propios desarrolladores de videojuegos para múltiples plataformas se han valido del arte urbano como un recurso visual y narrativo que, en la mayoría de los casos, es el eje vertebrador de toda la acción, mostrando un cierto grado de estudio centrado en la cultura urbana y, de forma generalizada, la visión criminalizada de este tipo de creaciones como es el caso de Jet Set Radio.

Considerando esto anterior, habría que sopesar la posibilidad de realizar un acercamiento pormenorizado a la iconografía subyacente en el arte urbano en torno a los videojuegos para analizar la relevancia discursiva que pudieran tener tanto para el artista como para el espectador y el entorno en el que se encuentra. Además, y en paralelo a este estudio sobre este interés del arte urbano por el mundo del juego, sería interesante contar con información en tanto a las opiniones de los artistas sobre los múltiples simuladores citados, para conocer hasta qué punto el mundo tecnológico se ha volcado en los intereses de los artistas y saber qué esperan de esta, cada vez más latente, vinculación entre arte y videojuego.

\section{Referencias:}

[1] ROJAS, M., "El boom de las gafas de realidad virtual en 2016", en GadgetoWeb. http://www.gadgetoweb.com/elboom-de-las-gafas-de-realidad-virtual-en-2016/[Consulta: 8/5/2016]

[2] NOQUEDANMASNICK, "Marc Ecko achaca la polémica de su juego a las diferencias intergeneracionales", en MeriStation (22-2-2006). http://www.meristation.com/noticias/marcecko-achaca-la-polemica-de-su-juego-a-las-diferenciasintergeneracionales/1637128 [Consulta: 12/10/2016]

[3] GRANDíO, P., "DJ Jota Mayúscula doblará al protagonista de Getting Up", en Vandal (13-7-2005). http://www.vandal.net/ noticia/17452/dj-jotamayuscula-doblara-al-protagonista-degetting-up/ [Consulta: 12/10/2016]

[4] GÓMEZ VILLAFAÑA, A. I., "El graffiti como fuente de inspiración para la industria de la moda", en Moda en el diseño (27-5-2015). http://modaeneldiseno.blogspot.com.es/2015/05/el-graffiticomo-fuente-de-inspiracion.html [Consulta: 1/8/2016]

[5] VÁZQUEZ DE LA FUENTE, M., "Vinculados a Ano", en Mural Street Art Conservation, № 3, pág. 52. https://issuu. com/observatoriodearteurbano/docs/mural_3 [Consulta: 4/8/2016] 
[6] AUGMENTED REALITY TRENDS, "Max Morrison- Augmented Reality Graffiti Creator". Traducido de Augmented Reality Trends (3-11-2014). http://www.augmentedrealitytrends.com/ ar-app/max-morrison.html [Consulta: 4/8/2016]

[7] MACDOWALL, L., "For lovers of graffiti, Pokemon Go is old hat", en The Conversation (19-7-2016). https://theconversation. com/for-lovers-of-graffiti-pokemon-go-is-old-hat-62683 [Consulta: 19/09/2016]

\section{Bibliografía:}

VÁZQUEZ DE LA FUENTE, M. (2016): Vinculados a Ano. Mural Street Art Conservation. Revista especializada digital del Observatorio de Arte Urbano №2. (pp. 52)

\section{Vínculos Web:}

AUGMENTED REALITY TRENDS, Max Morrison- Augmented Reality Graffiti Creator (3-11-2014). http://www. augmentedrealitytrends.com/ar-app/max-morrison.html (4-82016)
GÓMEZ VILLAFAÑA, A.I. El graffiti como fuente de inspiración para la industria de la moda. Moda en el diseño (27-5-2015). http://modaeneldiseno.blogspot.com.es/2015/05/el-graffiticomo-fuente-de-inspiracion.html (1-8-2016)

GRANDÍO, P., DJ Jota Mayúscula doblará al protagonista de Getting Up. Vandal (13-7-2005). http://www.vandal.net/ noticia/17452/dj-jotamayuscula-doblara-al-protagonista-degetting-up/ (12-10-2016)

MACDOWALL, L., For lovers of graffiti, Pokemon Go is old hat. The Conversation (19-7-2016). https://theconversation.com/forlovers- of-graffiti- pokemon-go- is-old- hat-62683 (19-9-2016)

NOQUEDANMASNICK., Marc Ecko achaca la polémica de su juego a las diferencias intergeneracionales. Meristation. (22-2-2006). http://www.meristation.com/noticias/marcecko-achaca-la-polemica-de-su-juego-a-las-diferenciasintergeneracionales/1637128 (12-10-2016)

ROJAS, M., El boom de las gafas de realidad virtual en 2016. Gadgetoweb. http://www.gadgetoweb.com/el-boom-de-lasgafas-de-realidad-virtual-en-2016/ (8-5-2016)

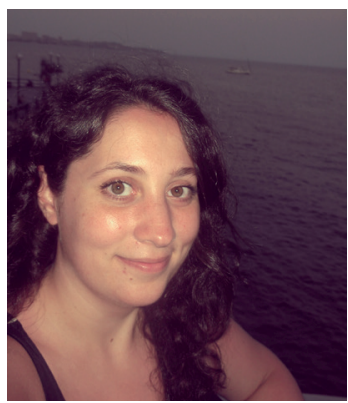

\section{María del Mar Vázquez de la Fuente}

Historiadora del arte

marimarvz@gmail.com

Graduada en Historia del Arte por la UAM (Universidad Autónoma de Madrid). Técnico en Radiografiado de Obras de Arte. 